\title{
Prevalence and prognostic and predictive relevance of PRAME in breast cancer
}

\author{
Padraig Doolan · Martin Clynes · Susan Kennedy • \\ Jai Prakash Mehta $\cdot$ John Crown · Lorraine O'Driscoll
}

Received: 1 June 2007 / Accepted: 1 June 2007/Published online: 12 July 2007

(C) Springer Science+Business Media B.V. 2007

\begin{abstract}
Preferentially expressed antigen of melanoma (PRAME) has been described as a potential candidate for immunotherapeutic targeting. However, the prognostic and predictive relevance of PRAME in breast cancer has never been investigated. PRAME gene expression was evaluated in 103 breast tumour biopsies, using quantitative reversetranscriptase polymerase chain reaction (qRT-PCR). Normal breast tissue was also analysed for comparative purposes. All qRT-PCRs were performed in triplicate. Kaplan-Meier survival curves, Chi-squared and Cox Regression analyses were used to identify associations between PRAME expression and patients' clinicopathological and survival data. PRAME mRNA was detected in $-53 \%$ of tumour specimens and $37 \%$ of normal breast specimens. Kaplan-Meier analysis showed expression of PRAME to correlate significantly with unfavourable disease outcome for patients, in terms of both their diseasefree survival $(p=0.0004)$ and overall survival (OS) $(p=0.0052)$ times from diagnosis. Multivariate analysis indicated PRAME expression to be an independent prognostic factor for shortened disease-free survival $(p=0.026)$ and $\mathrm{OS}(p=0.02)$. Furthermore, for patients who received adjuvant chemotherapy, significantly $(p=0.0291)$ shorter relapse-free survival was achieved for those whose tumour expressed PRAME, compared to those
\end{abstract}

Padraig Doolan and Martin Clynes contributed equally to this work.

P. Doolan · M. Clynes $\cdot$ J. P. Mehta $\cdot$ J. Crown .

L. O'Driscoll ( $\square)$

National Institute for Cellular Biotechnology, Dublin City

University, Dublin 9, Ireland

e-mail: Lorraine.ODriscoll@dcu.ie

S. Kennedy $\cdot$ J. Crown

St. Vincent's University Hospital, Dublin 4, Ireland that did not express this transcript. Our results suggest that PRAME mRNA expression may be a useful prognostic and predictive marker for breast cancer.

Keywords Breast cancer - PRAME - Disease/relapse-free survival · Overall survival - Quantitative reversetranscriptase polymerase chain reaction (qRT-PCR)

\section{Introduction}

Preferentially expressed antigen of melanoma (PRAME) was first described by Ikeda et al. [1] as a tumour antigen recognised by an autologous cytolytic $\mathrm{T}$ lymphocyte clone on a melanoma cell line. PRAME encodes a 509 amino acid protein and, although its function has not yet been defined, PRAME is reported to be a dominant repressor of retinoic acid receptor (RAR) signalling, inhibiting RAinduced differentiation, growth arrest and apoptosis [2-3].

Analysis of tumour and normal tissues by Northern blotting and RT-PCR indicated PRAME to be expressed by most melanomas ( $97 \%$ ), neuroblastomas (93\%), sarcomas $(\sim 80 \%)$, lung squamous cell carcinomas and small-cell lung cancers $(\sim 70 \%)$, Wilms' tumours [5], as well as many acute leukaemias (AML M3, 75\%; ALL, 64\%; CML BC $50 \%$; and AML M2 45\%). Intermediate frequencies of PRAME expression have been detected in renal cell carcinomas $(40 \%$; [4]), head and neck cancers $(29 \%)$, as well as adrenal, ovary and endometrial tissues, with low levels of expression present in many other normal tissue types $[1,5-7]$.

The PRAME has been proposed as a candidate for tumour immunotherapy, as it contains an immunogenic nonapeptide able to elicit a cytotoxic response when presented by HLA-A24 [1]. The relevance of PRAME as a 
candidate for immunotherapy was supported by recent studies indicating PRAME protein to be an antigen localised in the cell membrane, as well as in the cytoplasm, of cells [8].

As described above, PRAME is frequently expressed in a variety of cancers. However, with the exception of the study by Ikeda et al. [11] reporting PRAME mRNA to be expressed by $27 \%$ mammary carcinomas, no further information on its presence or relevance in breast cancer is available. This study investigated PRAME expression in 103 breast tumours and evaluated its association with clinical outcome.

\section{Materials and methods}

\section{Patient selection}

Tissue specimens from 103 cases of primary breast cancer (prior to any treatment with tamoxifen or chemotherapeutic agents), on which clinical follow-up and pathologic material, including snap-frozen tissue, were available for analysis from the 1993-1997 files of St. Vincent's University Hospital (SVUH) Pathology Department, Dublin, Ireland, were included in this study. Specimens were processed following approval from SVUH Ethics Committee and with patients' informed consent. The patients involved underwent potentially curative resection at the hospital. Unfixed fresh breast resection specimens were received in Pathology Laboratory within maximum $30 \mathrm{~min}$ of resection. The tissue was examined macroscopically and samples of tumour snap frozen in liquid nitrogen and subsequently stored at $-70 /-80^{\circ} \mathrm{C}$ prior to RNA isolation. A number of clinical and pathologic parameters were obtained from patients' charts including details on age, postoperative treatment and follow-up, tumour stage and hormonal analysis. Pathologic material was examined on each case by SK. Tumours were typed [9] and graded [10] as previously described. Staging was performed according to the TNM system of the UICC [11]. Nineteen non-cancerous breast biopsies were also included in these studies to represent normal breast tissue. A summary of the analysis involved in this study is illustrated in Fig. 1.

\section{RNA extraction}

For RNA analyses, dissected tumours that had been snapfrozen in liquid nitrogen and then stored at $-70 /-80^{\circ} \mathrm{C}$ until required were homogenised, on ice, in $2 \mathrm{ml}$ TriReagent (Sigma, Poole, UK) and total RNA was subsequently isolated according to the manufacturer's instructions. RNA quantity and purity were assessed at 260 and $280 \mathrm{~nm}$ using a Nanodrop (ND-1000; Labtech. International, East Sussex,
$\mathrm{UK}$ ); the $\mathrm{A}_{260} / \mathrm{A}_{280}$ ratio of pure RNA is $\sim 2$. RNA qualitatively was evaluated using an Agilent bioanalyser (Agilent 2100; Agilent Technologies, Santa Clara, CA, USA).

cDNA formation on mRNA template

Following priming with oligo (dT) at $65^{\circ} \mathrm{C}$ for $5 \mathrm{~min}$, followed by 1 min incubation on ice, cDNA was synthesised from $100 \mathrm{ng}$ total RNA, using Superscript III RNase H- (with increased thermal stability; Invitrogen), RNase OUT Ribonuclease (active against RNase A, B and C; Invitrogen) and a cocktail of dNTPs, by incubating at $50^{\circ} \mathrm{C}$ for $1 \mathrm{~h}$, followed by $70^{\circ} \mathrm{C}$ for $15 \mathrm{~min}$, in a $40 \mu \mathrm{l}$ reaction volume.

\section{Primer design}

Primers and TaqMan probes were designed using Primer Express Software 2.0 ensuring that, for optimum amplification efficiency, PCR primers produce an amplicon of $50-150 \mathrm{bp}$; flanking the probe, without overlap; with no more than two Gs and/or Cs at the $3^{\prime}$ end; and with a $T_{\mathrm{m}}$ of $58-60^{\circ} \mathrm{C}$. The probe designed to have a $\mathrm{G} / \mathrm{C}$ content in the $30-80 \%$ range, with no runs of four or more identical nucleotides; avoiding a $\mathrm{G}$ at the $5^{\prime}$ end; and with $T_{\mathrm{m}}$ values between 68 and $70^{\circ} \mathrm{C}$. Primers and dual-labelled probes, with 6-carboxyfluorescein (FAM) as reporter dye and 6-carboxy-tetramethyl-rhodamine (TAMRA) as quencher, were prepared by MWG.

\section{Quantitative reverse-transcriptase PCR (qRT-PCR)}

The cDNA (diluted 1:10), was amplified in $25 \mu$ reactions by quantitative PCR (qRT-PCR), using an ABI 7500 RealTime PCR System (Applied Biosystems, Foster City, CA, USA). Following evaluation of 12 potential endogenous controls, including $18 \mathrm{~S}$, acid ribosomal protein, $\mathrm{B}_{2}$-microglobulin, $\beta$-actin, cyclophilin, GAPDH, phosphoglycerokinase, $\beta$-glucronidase, hypoxanthine, ribosyl transferase, transcription factor IID and transferring receptor (Applied Biosystems; 430992) in a random selection of 14 breast specimens (seven breast tumours and seven normal breast specimens), this study involved evaluation, in all 103 breast carcinomas and 19 normal breast specimens, of PRAME mRNA. Primer and probes sequences for PRAME amplification were as follows: forward primer $-5^{\prime}$ ACTGCTCCCAGCTTACAACCTTA $3^{\prime}$ (length $=23 \quad T_{\mathrm{m}}=58$; $\% \mathrm{GC}=48$ ); reverse primer $-5^{\prime}$ TACAGCACGTGGGTCAGATTG $3^{\prime} \quad$ (length $=21 ; \quad T_{\mathrm{m}}=58 ; \quad \% \mathrm{GC}=52$ ); probe-ATCTCCATATCTGCCTTGCAGAGTCTCCTG (length $=30 ; T_{\mathrm{m}}=68 ; \% \mathrm{GC}=50$ ), resulting in an amplified product of $112 \mathrm{bp}$. The temperature profile of all reactions was $50^{\circ} \mathrm{C}$ for $2 \mathrm{~min}, 95^{\circ} \mathrm{C}$ for $10 \mathrm{~min}, 40$ cycles of 
Fig. 1 Summary of analysis work-flow

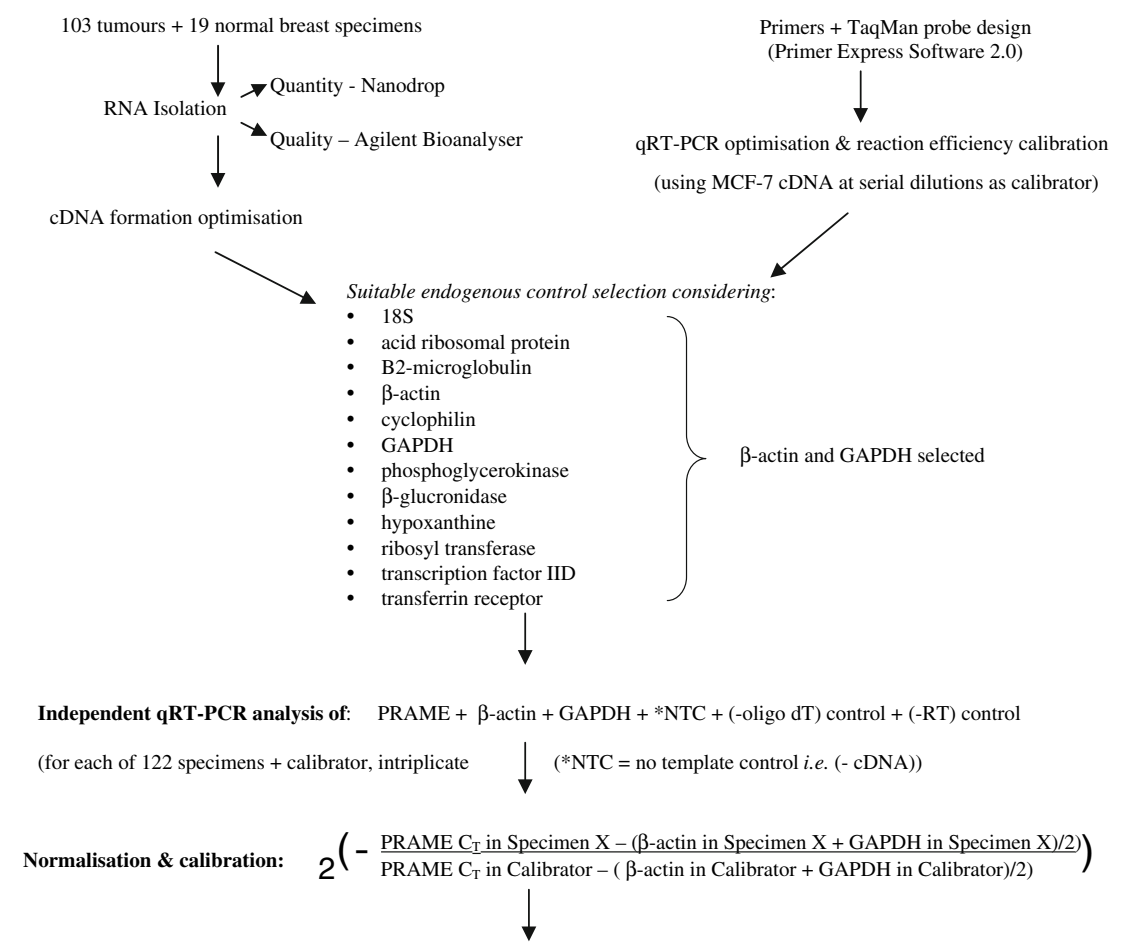

Statistical (univariate and multivariate) analyses: of expression association with clinicopathological characteristics and patients' outcome
95 and $60^{\circ} \mathrm{C}$ for $1 \mathrm{~min}$. Individual specimens were analysed in triplicate, with absence of cDNA samples included as negative controls. Minus reverse transcriptase enzyme and minus oligo(dT) controls verified no DNA/pseudogene contamination of starting material. PRAME threshold cycle $\left(C_{\mathrm{T}}\right)$ results were subsequently normalised to two suitable endogenous controls $-\beta$-actin and GAPDH-and calibrated against MCF-7 cDNA using the comparative $C_{\mathrm{T}}$ method, $2^{-\Delta \Delta \mathrm{CT}}[12]$. Subsequently, $2^{-\Delta \Delta \mathrm{CT}}$ values of $<500$ were considered as absent/base-line, while $\geq 500$ levels were considered as positive expression of PRAME.

\section{Statistical analysis}

Statistical (univariate and multivariate) analyses of the results were performed using the SPSS 10.1 software package. Descriptive statistics were used to summarise patient characteristics and statistical analysis of the results was performed using Pearson's $\chi^{2}$-test to investigate relationships between mRNA expression and clinicopathological and histopathologic findings. Kaplan-Meier survival curves were established and were subsequently checked using the log-rank, Breslow and Tarone-ware tests ( $p$-values represent log-rank, unless otherwise indicated) to assess the prognostic and predictive significance of expression of analysed mRNAs in tumour cells. Multivariate survival analyses were performed using the Cox regression backward stepwise likelihood ratio. The data was censored at 5 years for multivariate analysis. $p<0.05$ was considered statistically significant.

\section{Results}

\section{Patient characteristics}

This study involved analysis of 103 breast cancer biopsies (removed prior to any treatment with tamoxifen or chemotherapeutic agents) from patients aged between 31 and 90 years at the time of diagnosis (mean age 58 years). Twenty-six women were $<50$ years and 77 women were 50 years, or older, at diagnosis.

The size of the tumours ranged between 0.6 and $8.0 \mathrm{~cm}$ (mean $2.8 \mathrm{~cm}$ ). Eighteen tumours were $\mathrm{T} 1(<2 \mathrm{~cm})$ in maximal dimension; 82 tumours were T2 $(2-5 \mathrm{~cm})$ and three tumours were T3 $(>5 \mathrm{~cm})$. Eighty-one tumours were invasive ductal carcinoma, 17 were invasive lobular and five were tumours of special type (two tubular and three mucinous). Eleven tumours were grade 1; 39 were grade 2; and 53 were grade 3 . Sixty-six tumours were oestrogen receptor positive and 34 were oestrogen receptor negative (oestrogen receptor status was determined by Enzyme Immuno-Assay (EIA); a positive result was defined as more than $200 \mathrm{fmol} / \mathrm{g}$ protein). Oestrogen receptor status was not available for three patients. Forty-five tumours had no axillary metastases and 58 tumours had metastasised to axillary lymph nodes. 
Sixty-nine women were treated with post-operative tamoxifen; 25 did not receive tamoxifen. Forty-nine patients were treated with adjuvant systemic chemotherapy (CMF +/- adriamycin); 45 patients did not receive chemotherapy. Details regarding tamoxifen and systemic chemotherapy were not available for nine patients. Maximal follow-up was 3,026 days with a mean follow-up of 1,887 days.

\section{Detection of PRAME mRNA}

The PRAME mRNA was detected in 53.4\% (55/103) of the breast tumour specimens analysed [and in $36.8 \%$ (7/19) of the normal breast specimens].

Prognostic analysis of PRAME for disease-free survival

Cox univariate analysis (Table 1) indicated that expression of PRAME associated significantly $(p=0.001)$ with relapse-free survival (RFS). Tumour grade $(p<0.0005)$ and size $(p=0.009)$, treatment with adjuvant chemotherapy ( $p=0.002)$, lymph node status $(p<0.0005)$ and ER status $(p=0.004)$ also showed significant correlation with RFS. These results were supported by Kaplan-Meier analysis (Table 1), which indicated that ER positivity to be a favourable factor $(p=0.0032)$, while PRAME expression $(p=0.0004)$, increased tumour size $(p=0.007)$, advanced grade $(p=0.0013)$, treatment with adjuvant chemotherapy $(p=0.0012)$ and spread to lymph nodes $(p<0.00005)$ all correlated significantly with bad prognosis. As detailed in Table 2, chi-squared analysis indicated no significant associations between PRAME mRNA expression and other clinicopathological characteristics. By multivariate analysis (Table 3) independent prognostic factors for RFS were found to be lymph node status $(p=0.007)$, tumour grade $(p=0.013), \quad$ tumour size $\quad(p=0.005), \quad$ ER status $(p=0.015)$ and PRAME mRNA expression $(p=0.026)$. Kaplan-Meier analysis indicating a significant unfavourable association between RFS and expression of PRAME $(p=0.0004)$ is shown in Fig. 2.

Prognostic analysis of PRAME for overall survival

Univariate Cox analysis (Table 1) indicated PRAME mRNA expression to be a significant $(p=0.007)$ prognostic indicator of overall survival (OS). Other factors investigated in this study which were found to be significantly associated with OS include tumour size $(p=0.048)$, tumour grade $(p<0.0005)$, tumour type $(p=0.031)$ and lymph node status $(p=0.001)$. These results were supported by Kaplan-Meier analysis, indicating PRAME expression $(p=0.0052)$, increased size $(p=0.0437)$, advanced stage $(p=0.0003)$ and type $(p=0.0453)$ of tumours and spread to lymph nodes $(p=0.0006)$ significantly correlated with poor prognosis. No significant association was found between OS and age at diagnosis, ER status ( $p=0.05$; so approaching significance) or adjuvant tamoxifen therapy. Figure 2 illustrates significant unfavourable $(p=0.0052)$ association between PRAME expression and OS by Kaplan-Meier analysis. Multivariate analysis (Table 3) indicated tumour grade $(p=0.014)$, tumour size $(p=0.023)$, ER status $(p=0.035)$ and PRAME expression $(p=0.02)$ to be independent prognostic factors for OS.

\section{Predictive relevance of PRAME expression}

To establish if expression of PRAME mRNA is predictive of response to adjuvant chemotherapy, Kaplan-Meier

Table 1 Univariate Cox analysis supported by Kaplan-Meier analysis

\begin{tabular}{|c|c|c|c|c|}
\hline \multirow[t]{2}{*}{ Characteristics } & \multicolumn{2}{|c|}{ Overall survival (OS) } & \multicolumn{2}{|c|}{ Relapse-free survival (RFS) } \\
\hline & $p^{\mathrm{a}}$ & $p^{\mathrm{b}}$ & $p^{\mathrm{a}}$ & $p^{\mathrm{b}}$ \\
\hline Age $(<50$ versus $\geq 50$ years $)$ & 0.964 & 0.9636 & 0.263 & 0.2599 \\
\hline Tumour size $(<2.8$ versus $\geq 2.8 \mathrm{~cm})$ & $0.048^{*}$ & $0.0437 *$ & $0.009 *$ & $0.007 *$ \\
\hline Lymph node metastasis (negative versus positive) & $0.001^{*}$ & $0.0006^{*}$ & $<0.0005^{*}$ & $<0.00005^{*}$ \\
\hline Histology grade (I and II versus III) & $<0.0005^{*}$ & $0.0003^{*}$ & $<0.0005^{*}$ & $0.0013 *$ \\
\hline Histology type (IDC versus ILC versus special) & $0.031^{*}$ & $0.0453 *$ & 0.206 & 0.0624 \\
\hline ER status (negative versus positive) & 0.056 & 0.0514 & $0.004 *$ & $0.0032 *$ \\
\hline Chemotherapy (yes versus no) & 0.050 & $0.0457 *$ & $0.002 *$ & $0.0012 *$ \\
\hline Tamoxifen (yes versus no) & 0.449 & 0.4475 & 0.182 & 0.1784 \\
\hline PRAME & $0.007^{*}$ & $0.0052 *$ & $0.001 *$ & $0.0004 *$ \\
\hline
\end{tabular}

* Significant parameter - ${ }^{\mathrm{a}}$ denoted Cox regression $p$-value, ${ }^{\mathrm{b}}$ indicates Kaplan-Meier $p$-value; mean size (2.8 cm) was used as cut-off; grades I and II were grouped together versus grade III; IDC invasive ductal carcinoma, ILC invasive lobular carcinoma. Kaplan-Meier analysis support the univariate Cox regression studies, indicating ER positivity to be a favourable prognostic factor, with PRAME mRNA expression, increased tumour size, tumour type, advanced grade, spread to nodes, and adjuvant chemotherapy treatment associated with poor outcome for patients 
Table 2 Correlation between clinicopathological factors and expression of PRAME mRNA in breast carcinoma

\begin{tabular}{|c|c|c|c|}
\hline Characteristics & Number of cases & PRAME $500(\%)$ & $p$ \\
\hline \multicolumn{4}{|l|}{ Age (years) } \\
\hline$<50$ & $14 / 26$ & 53.8 & \multirow[t]{2}{*}{0.958} \\
\hline$\geq 50$ & $41 / 77$ & 53.2 & \\
\hline \multicolumn{4}{|l|}{ Tumour size } \\
\hline $\mathrm{T} 1(<2 \mathrm{~cm})$ & $9 / 18$ & 50.0 & \multirow[t]{3}{*}{0.726} \\
\hline $\mathrm{T} 2(2-5 \mathrm{~cm})$ & $45 / 82$ & 54.9 & \\
\hline $\mathrm{T} 3(>5 \mathrm{~cm})$ & $1 / 3$ & 33.3 & \\
\hline \multicolumn{4}{|c|}{ Lymph node metastasis } \\
\hline Negative & $20 / 45$ & 44.4 & \multirow[t]{2}{*}{0.109} \\
\hline Positive & $35 / 58$ & 60.3 & \\
\hline \multicolumn{4}{|l|}{ Histology grade } \\
\hline I & $5 / 11$ & 45.5 & \multirow[t]{3}{*}{0.556} \\
\hline II & $19 / 39$ & 48.7 & \\
\hline III & $31 / 53$ & 58.5 & \\
\hline \multicolumn{4}{|l|}{ Histology type } \\
\hline IDC & $43 / 81$ & 53.1 & \multirow[t]{3}{*}{0.955} \\
\hline ILC & $9 / 17$ & 52.9 & \\
\hline Special & $3 / 5$ & 60.0 & \\
\hline \multicolumn{4}{|l|}{ ER status } \\
\hline Negative & $18 / 34$ & 52.9 & \multirow[t]{2}{*}{0.892} \\
\hline Positive & $34 / 66$ & 51.1 & \\
\hline \multicolumn{4}{|l|}{ Chemotherapy } \\
\hline No & $23 / 45$ & 51.1 & \multirow[t]{2}{*}{0.234} \\
\hline Yes & $31 / 49$ & 63.3 & \\
\hline \multicolumn{4}{|l|}{ Tamoxifen } \\
\hline No & $13 / 25$ & 52.0 & \multirow[t]{2}{*}{0.697} \\
\hline Yes & $39 / 69$ & 56.5 & \\
\hline
\end{tabular}

$p$-values from $\chi^{2}$ analyses; IDC invasive ductal carcinoma, ILC invasive lobular carcinoma

analysis was performed on those cases only where chemotherapy was administered. As indicated in Fig. 3, while PRAME expression was not predictive of OS for this group of patients, the results of this analysis suggest PRAME expression to be predictive of poorer outcome in terms of RFS ( $p=0.0291)$, with expression of PRAME associated with shortened time to relapse.

\section{Discussion}

In breast cancer, prognostic factors currently relied upon are those determined by clinical or standard pathological approaches namely lymph node status, tumour size, histological grade, nuclear grade and tumour histology. Predictive factors are limited to ER, progesterone receptor, and her $2 / n e u$, which are used to predict response to hormonal treatment and herceptin, respectively; no reliable
Table 3 Multivariate Cox regression backward stepwise (likelihood ratio)

\begin{tabular}{lll}
\hline Characteristics & $\begin{array}{l}\text { Overall survival } \\
\text { (OS) } \\
p\end{array}$ & $\begin{array}{l}\text { Relapse-free } \\
\text { survival (RFS) } \\
p\end{array}$ \\
\hline $\begin{array}{l}\text { Lymph node (spread versus } \\
\text { no spread) }\end{array}$ & NS (0.060) & 0.007 \\
$\begin{array}{l}\text { ER (absence versus } \\
\text { presence) }\end{array}$ & 0.035 & 0.015 \\
$\begin{array}{l}\text { Histology grade (I and II } \\
\text { versus III) }\end{array}$ & 0.014 & 0.013 \\
$\begin{array}{l}\text { Tumour size (<2.8 versus } \\
\geq 2.8 \text { cm) }\end{array}$ & 0.023 & 0.005 \\
PRAME mRNA & 0.020 & 0.026 \\
\hline
\end{tabular}

Parameters included in the multivariate analysis included age, tumour size, tumour grade, lymph node status, ER status, as well as PRAME mRNA expression. Mean size $(2.8 \mathrm{~cm})$ was used as cut-off; grades I and II were grouped together versus grade III. This table summarises significant factors

$N S$ not significant

predictive markers for response to chemotherapy have been identified. A more extensive range of prognostic and predictive markers is needed in order to extend the range of individualised therapies for breast cancer patients. The study presented here represents the first reported analysis of the relevance, to breast cancer patients, of PRAME expression. Using qRT-PCR analysis of 103 breast tumours patients, PRAME mRNA expression prevalence and relevance to outcome for patients, over a mean follow-up period of 1,887 days (maximum follow-up of 3,026 days) post-diagnosis has been evaluated.

The PRAME mRNA was detected in $~ 53 \%$ of the breast tumours and $\sim 37 \%$ of normal breast biopsies analysed. The percentage positivity reported in breast tumours here (i.e. $53 \%$ ) is somewhat higher than that found in the only previous study of PRAME in mammary carcinomas where, using RT-PCR, $27 \%$ of specimens were reported to express this transcript [1]. Potential reasons from this discrepancy may be differences in methods of analysis, for example, the increased sensitivity of qRT-PCR and the method used by Ikeda to score PRAME positivity, i.e. in their study samples were scored positive if their expression of PRAME gene exceeded 3\% of that found in MEL.A cell line. Analysis of PRAME mRNA in normal breast tissue had not been investigated previously. Our findings are in keeping with reports of some PRAME expression present in many other normal tissue types [1,5-7].

Although the incidence of PRAME expression has been assessed in many cancer types by other researchers, the clinicopathological relevance of its expression has, in general, been limited to neuroblastoma, leukaemia and multiple myeloma. A study of 101 neuroblastoma cases 
Fig. 2 Kaplan-Meier survival curves for PRAME mRNA presence or absence and its association with disease/ relapse-free survival $(R F S)$ and overall survival $(O S)$, respectively
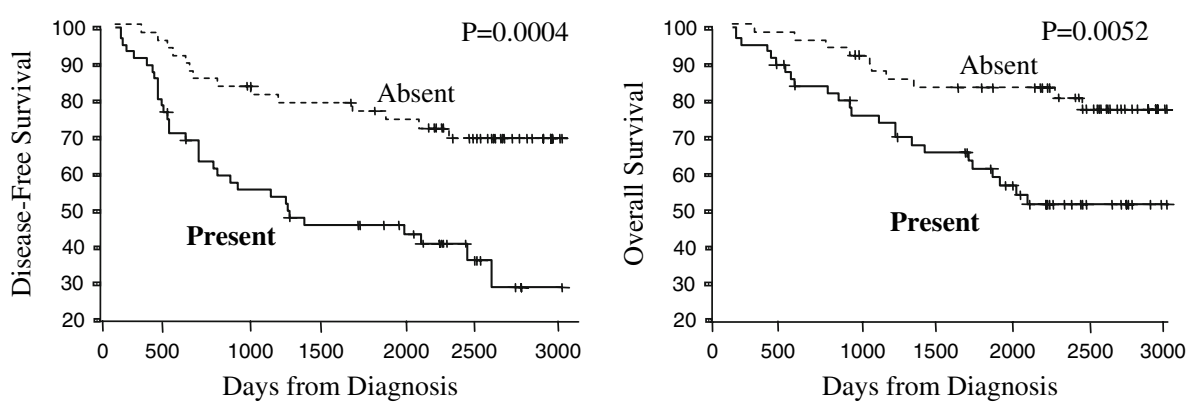

Fig. 3 Kaplan-Meier survival curves for PRAME mRNA presence or absence and its association with disease/ relapse-free survival $(R F S)$ and overall survival $(O S)$, in cases when adjuvant chemotherapy was administered
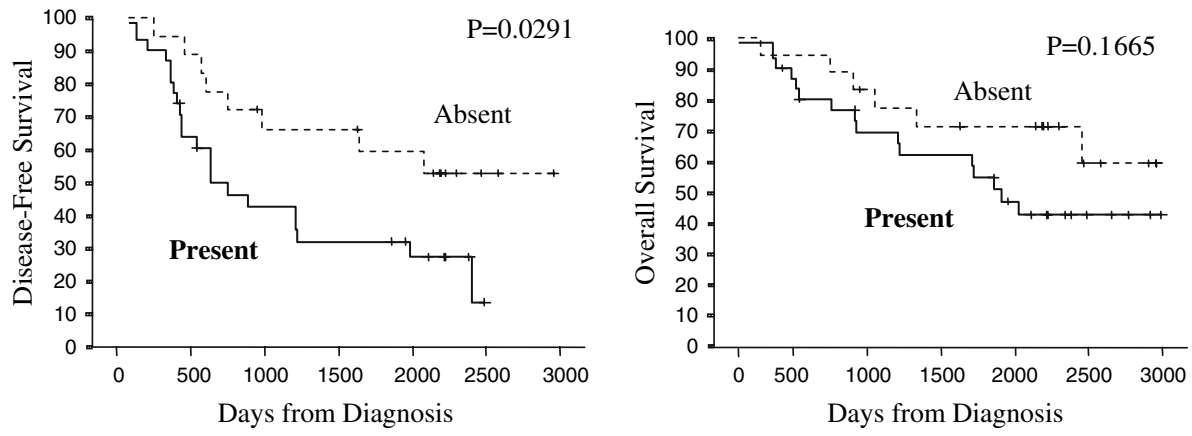

(using Northern blotting, RT-PCR or qRT-PCR) indicated PRAME expression in $93 \%$ of primary neuroblastoma and $100 \%$ of patients with advanced disease. Results from RT-PCR and Northern blotting analysis indicated a significant association between PRAME expression and both patients' age at diagnosis and higher tumour stage, while analysis of qRT-PCR data at three intervals (those $<100$, $100-20,000$ and $>20,000$ ) of expression level indicated higher expression levels to be associated with unfavourable outcome in terms of both disease-free survival (RFS/DFS) and OS [7]. As described previously, analysis of blood disorders has indicated that PRAME is transcribed in leukaemia cells, but not in normal bone marrow or peripheral blood mononuclear cells [13-14]. However, reports on the clinical relevance of PRAME expression in blood diseases have been conflicting. PRAME has been suggested as a specific marker for acute megakaryoblastic leukaemia, with no expression of this mRNA detected in transient myeloproliferative disorder [15] and recent studies have concluded that PRAME quantification by qRTPCR appears to be suitable for monitoring minimal residual disease in PRAME-positive leukaemia [16, 17]. Studies of childhood acute myeloid leukaemia, however, suggest PRAME mRNA (found in $\sim 60 \%$ of cases) expression to be an indicator of favourable outcome, in terms of both RFS and OS [18]. Similarly, in studies of childhood acute lymphoblastic leukaemia, over-expression of PRAME mRNA [found in $42 \%(21 / 50)$ cases] was reported to be associated favourably with disease-free survival, although statistical significance was not achieved in this case [19]. In contrast, higher levels of PRAME expression are associated with advanced stages of multiple myeloma [20].

In our study of breast cancer, PRAME gene expression at diagnosis did not correlate with established clinical or pathologic characteristics namely, ER status of patients, lymph node status, histological sub-type, histological grade, tumour size or subsequent treatment with adjuvant chemotherapy. However, as with studies of multiple myeloma and neuroblastoma [7], we have found PRAME expression to be associated with unfavourable outcome for breast cancer patients-with a significant correlation between expression of this transcript in breast tumours and shortened RFS and OS from diagnosis. Indeed, multivariate analysis indicates that, similarly to tumour grades, tumour sizes and ER status (i.e. ER-negativity), PRAME mRNA expression in breast tumours is an independent unfavourable prognostic factor for both RFS and OS.

With the exception of a study of leukaemias and lymphomas [21], the predictive relevance of PRAME expression in cancer had not yet been elucidated. Results from our study were thus analysed to investigate a potential predictive relevance for PRAME in breast cancer and a significant association was found between expression of this transcript and unfavourable outcome for patients who received adjuvant CMF-based chemotherapy, in terms of RFS. This finding of PRAME expression apparently predicting earlier relapse for breast cancer patients (compared to those whose tumour do not express PRAME) is broadly in agreement with the study of 98 patients with leukaemias and lymphomas [21], where significantly reduced levels 
of PRAME mRNA were found following chemotherapy administration, with increased expression detected in two patients who relapsed.

The findings reported here suggest that PRAME mRNA expression may have potential not only as a prognostic, but also as a predictive, biomarker for breast cancer. Future studies of larger cohorts of breast cancer and normal tissue, as well investigations of the prognostic and predictive relevance of PRAME in other cancer types, will help further define the potential of this biomarker as one of a panel of molecular targets for routine analysis in cancer.

Acknowledgements This work was supported by funding from Ireland's Higher Educational Authority Programme for Research in Third Level Institutions (PRTLI) Cycle 3, Dublin City University's Albert College Fellowship, Dublin City University's Research Fellowship and the Health Research Board.

\section{References}

1. Ikeda H, Lethe B, Lehmann F et al (1997) Characterization of an antigen that is recognized on a melanoma showing partial HLA loss by CTL expressing an NK inhibitory receptor. Immunity 6:199-208

2. Epping MT, Wang L, Edel MJ, Carlee L, Hernandez M, Bernards $\mathrm{R}$ (2005) The human tumour antigen PRAME is a dominant repressor of retinoic acid receptor signaling. Cell 122:835-847

3. Epping MT, Bernards R (2006) A causal role for the human tumour antigen preferentially expressed antigen of melanoma in cancer. Cancer Res 66:10639-10642

4. Neumann E, Engelsberg A, Decker J et al (1998) Heterogeneous expression of the tumour-associated antigens RAGE-1, PRAME, and glycoprotein 75 in human renal cell carcinoma: candidates for T-cell-based immunotherapies? Cancer Res 58:4090-4095

5. Li CM, Guo M, Borczuk A et al (2002) Gene expression in Wilms' tumour mimics the earliest committed stage in the metanephric mesenchymal-epithelial transition. Am J Pathol 160:2181-2190

6. Matsushita M, Yamazaki R, Kawakami Y (2004) Quantitative analysis of PRAME for detection of minimal residual disease in leukaemia. Methods Mol Med 97:267-275

7. Oberthuer A, Hero B, Spitz R, Berthold F, Fischer M (2004) The tumour-associated antigen PRAME is universally expressed in high-stage neuroblastoma and associated with poor outcome. Clin Cancer Res 10:4307-4313

8. Proto-Siqueira R, Figueiredo-Pontes LL, Panepucci RA et al (2006) PRAME is a membrane and cytoplasmic protein aberrantly expressed in chronic lymphocytic leukemia and mantle cell lymphoma. Leuk Res 30:1333-1339

9. Page DL, Andersen AT (1987) Diagnostic histopathology. Churchill Livingstone, London, Melbourne and New York

10. Elston CW, Ellis IO (1991) Pathological prognostic factors in breast cancer. I. The value of histological grade in breast cancer: experience from a large study with long-term follow-up. Histopathology 403-410

11. Sobin LH, Wittekind C (1997) TNM Classification of Malignant tumours. Wiley, New York

12. Livak KJ, Schmittgen TD (2001) Analysis of relative gene expression data using real-time quantitative PCR and the 2(-Delta Delta C(T). Methods 25:402-408

13. van Baren N, Chambost H, Ferrant A et al (1998) PRAME, a gene encoding an antigen recognized on a human melanoma by cytolytic $\mathrm{T}$ cells, is expressed in acute leukaemia cells. Br J Haematol 102:1376-1379

14. Watari K, Tojo A, Nagamura-Inoue $\mathrm{T}$ et al (2000) Identification of a melanoma antigen, PRAME, as a BCR/ABL-inducible gene. FEBS Lett 466:367-371

15. McElwaine S, Mulligan C, Groet J et al (2004) Microarray transcript profiling distinguishes the transient from the acute type of megakaryoblastic leukaemia (M7) in Down's syndrome, revealing PRAME as a specific discriminating marker. $\mathrm{Br} \mathrm{J}$ Haematol 125:729-742

16. Paydas S, Tanriverdi K, Yavuz S, Disel U, Baslamisli F, Burgut R (2005) PRAME mRNA levels in cases with acute leukemia: clinical importance and future prospects. Am J Hematol 79:257-261

17. Tajeddine N, Millard I, Gailly P, Gala JL (2006) Real-time RTPCR quantification of PRAME gene expression for monitoring minimal residual disease in acute myeloblastic leukaemia. Clin Chem Lab Med 44:548-555

18. Steinbach D, Hermann J, Viehmann S, Zintl F, Gruhn B (2002) Clinical implications of PRAME gene expression in childhood acute myeloid leukemia. Cancer Genet Cytogenet 133:118-123

19. Steinbach D, Viehmann S, Zintl F, Gruhn B (2002) PRAME gene expression in childhood acute lymphoblastic leukemia. Cancer Genet Cytogenet 138:89-91

20. van Baren N, Brasseur F, Godelaine D et al (1999) Genes encoding tumour-specific antigens are expressed in human myeloma cells. Blood 94:1156-1164

21. Matsushita M, Ikeda H, Kizaki M et al (2001) Quantitative monitoring of the PRAME gene for the detection of minimal residual disease in leukaemia. Br J Haematol 112:916-926 